\title{
Straightening out the wrinkles in technetium- 99m-labeled bone scintigraphy tracer assessment of cardiac amyloidosis
}

\author{
Gary R. Small, BSc, PhD, MB, ChB, MRCP, ${ }^{a}$ and Terrence D. Ruddy, MD, FRCP, \\ FACC, FAHA, FCCS ${ }^{a}$ \\ a Department of Cardiac Imaging, University of Ottawa Heart Institute, Ottawa, ON, Canada
}

Received Apr 2, 2019; accepted Apr 2, 2019

doi: $10.1007 / \mathrm{s} 12350-019-01718-8$

\section{See related article, pp. 90-99}

\section{INTRODUCTION}

Cardiac amyloidosis is characterized by the abnormal deposition of misfolded proteins in the heart. ${ }^{1,2} \mathrm{~A}$ characteristic feature of amyloid proteins are the wrinkled B-pleated sheets formed within infiltrated tissues. In the majority of cases, cardiac amyloid can be attributed to light chain amyloid (AL) or transthyretin amyloid (ATTR) protein accumulation. ATTR may be further sub-classified as wild type (senile) or gene mutation (familial). ${ }^{2}$ Clinically, ATTR and AL can be similar and distinguishing between them may be a challenge. Scintigraphy using technetium-99m $\left({ }^{99 \mathrm{~m}} \mathrm{Tc}\right)$ labeled bone tracers is helpful in detecting cardiac amyloid and may help differentiate between ATTR and AL cardiac amyloid.

ATTR cardiac amyloid typically demonstrates avid myocardial uptake of ${ }^{99 \mathrm{~m}}$ Tc-labeled bone tracers, whereas in AL cardiac amyloid, cardiac uptake if present, is characteristically low. On occasion however a cardiac amyloid SPECT study using a ${ }^{99 \mathrm{~m}}$ Tc-labeled bone tracer may be equivocal for ATTR amyloid as the low myocardial uptake of tracer could represent either ATTR or AL amyloid. ${ }^{3}$

Current American Society of Nuclear Cardiology practice points for ${ }^{99 \mathrm{~m}} \mathrm{Tc}$-pyrophosphate imaging

Reprint requests: Gary R. Small, BSc, PhD, MB, ChB, MRCP, Department of Cardiac Imaging, University of Ottawa Heart Institute, Ottawa, ON K1Y 4W7, Canada; gsmall@ottawaheart.ca J Nucl Cardiol 2021;28:100-3.

$1071-3581 / \$ 34.00$

Copyright (c) 2019 American Society of Nuclear Cardiology. suggest image acquisition at 1 hour after tracer injection and at 3 hours if significant blood pool is seen on the 1 hour images. ${ }^{3}$ Images are reviewed using a visual grading approach proposed by Perugini $e t a l^{4}$ and a semi-quantitative heart to contra-lateral lung (H/CL) ratio (Figure 1). ATTR amyloid is strongly suggested by a Perugini grade of $>1$ and a $\mathrm{H} / \mathrm{CL}$ ratio $>1.5$. Equivocal studies have $\mathrm{H} / \mathrm{CL}$ ratios of $1-1.5$ and/or Perugini grade of $0-1 .^{3}$

\section{SEMI-QUANTITATIVE ANALYSIS USING ${ }^{99}$ TC- HYDROXYMETHYLENE DIPHOSPHATE (HMDP)}

The inclusion of semi-quantitative analysis to the diagnostic protocol is a recent addition. ${ }^{3,5}$ Prior approaches for non-biopsy diagnosis of cardiac ATTR have been framed around the use of Perugini visual grading. Semi-quantitative analysis is an attractive goal for scintigraphic assessment of cardiac amyloid and can support the visual analysis. As well, semi-quantitative analysis may offer other benefits including interval disease monitoring, assessing treatment response, excluding disease with screening, and prognosis evaluation. ${ }^{6}$

In this issue of the Journal, Gallini et al present a comparison of the operating characteristics of six semiquantitative measures of cardiac amyloid uptake using heart to different-body-region ratios in 76 patients with suspected cardiac amyloid. ${ }^{7}$ In addition to comparing semi-quantitative indices, they determined whether any of the examined ratios may better discriminate equivocal scans with semi-quantitative grade of $0-1$ from scans with grade 2-3 visual scores to more reliably differentiate ATTR from AL cardiac amyloid.

All patients had ${ }^{99 \mathrm{~m}} \mathrm{Tc}-\mathrm{HMDP}$ whole body scintigraphy at 150 minutes following a $740 \mathrm{MBq}$ injection. Semi-quantitative analysis was performed comparing cardiac uptake with (a) skeletal regions (skull or pelvis) 


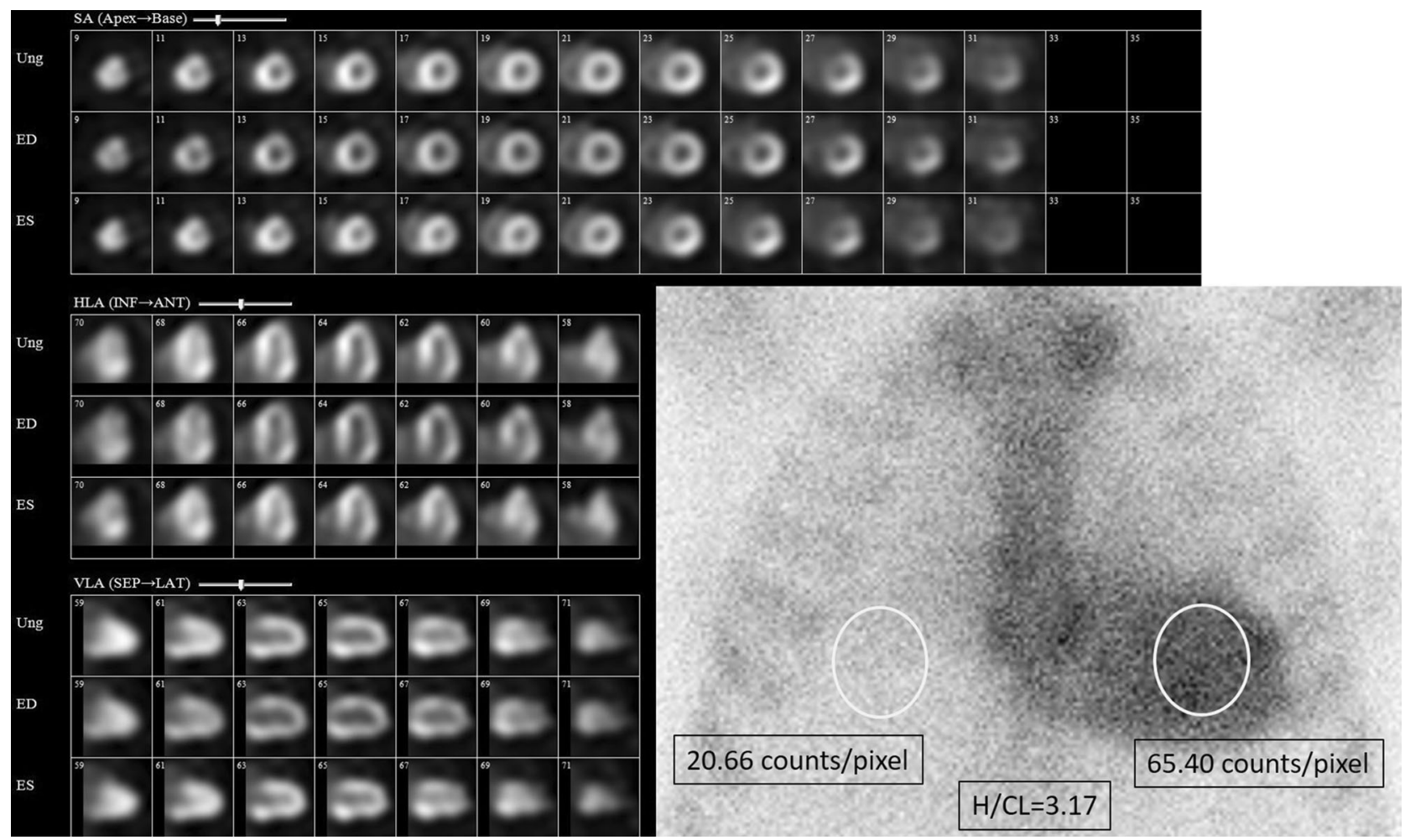

Figure 1. One hour ${ }^{99 \mathrm{~m}}$ Tc-pyrophosphate planar and SPECT imaging a patient with biopsy positive wild-type ATTR cardiac amyloid. Perugini grade 3 uptake on visual scoring. Heart/contralateral lung ratio 3.17 .

(b) the whole body (H/WB) or (c) heart to contra-lateral lung $(\mathrm{H} / \mathrm{CL})$ ratios. Visual comparison of heart to rib uptake was made using the Perugini approach (cardiac uptake being graded as $0=$ no uptake, $1=$ less than rib uptake, $2=$ similar to rib uptake, and $3=$ greater than rib uptake). ${ }^{4}$ Of the 76 study patients, ATTR cardiac amyloid was found in 53, AL amyloid in 12 and nonamyloid related left ventricular hypertrophy (LVH) in 11 patients.

Consistently, elevated ratios of heart vs the different body regions were found in patients with ATTR vs the other patient groups. These findings were also noted using a novel ratio of heart/pelvis presented for the first time in this report. The highest ratios were seen with heart/whole body $(\mathrm{H} / \mathrm{WB})$ ratios $(6.0 \pm 1.6)$. Using a $\mathrm{H} /$ WB ratio of $3.28,100 \%$ sensitivity and specificity were observed for ATTR amyloid. A ratio of 1.37 for H/CL demonstrated a sensitivity of $98 \%$ and specificity of $100 \%$ for ATTR amyloid. One false negative occurred using $\mathrm{H} / \mathrm{CL}$ ratio due to lung uptake of ${ }^{99 \mathrm{~m}} \mathrm{Tc}-\mathrm{HMDP}$. The novel parameter of $\mathrm{H} / \mathrm{P}$ ratio performed well with a specificity of $100 \%$ and sensitivities of $\geq 95 \%$.

Statistically different $\mathrm{H} / \mathrm{WB}$ ratios were seen between Perugini scores of 1 and 2 (Table 3 in the paper). Thus, H/WB semi-quantitative ratios could help to better differentiate those patients with AL and ATTR cardiac amyloid when the Perugini scores are 1.

\section{WHICH ${ }^{99 M}$ TC-LABELED BONE TRACER IS BEST?}

Several ${ }^{99 \mathrm{~m}}$ Tc-labeled bone tracers have been used to assess ATTR cardiac amyloid including HMDP, 3,3diphosphono-1,2-propanodicarboxylic acid (DPD), and pyrophosphate (PYP). ${ }^{8}$ These tracers demonstrate retention in myocardial scar following myocardial infarction although the distribution in such circumstances will be restricted to the coronary territory involved. There are several suggested bindings sites for the tracers including amyloid microcalcifications, calcium deposits, intracellular calcium or intracellular macromolecules. ${ }^{2}$ Both ATTR and AL amyloid have areas of microcalcification on endomyocardial biopsy. Microcalcification was more abundant in cases of ATTR amyloid and may explain the more avid tracer retention in ATTR vs AL cardiac amyloid. $^{9}$

No study has compared the performance of all three tracers probably due to the regional variations in availability of the radiotracers. Although in Italy both DPD and HMDP are available, local health service buying policies have restricted certain regions to 
HMDP. ${ }^{7}$ PYP is available in North America and DPD is used in other European centers. Discerning the merits of one tracer over another is therefore challenging. In a collaborative paper considering the non-biopsy diagnosis of cardiac amyloid that included British, European and US centers, some observations are possible regarding the performance of all three radiotracers although the patients were not cross tested with each tracer. ${ }^{5}$ HMDP scintigraphy was performed in 18 cases of biopsy proven cardiac amyloid and no cases of $\mathrm{AL}$ amyloid were noted to have a Perugini score of $>0$. In contrast within both DPD and PYP imaged cohorts, cases of AL amyloid were noted to have Perugini grades of 0-3. The cohorts of these patients were much larger, 244 patients for DPD and 109 for PYP. So although HMDP may appear to be more selective for ATTR than DPD or PYP, the small numbers of patients imaged in this study with HMDP make drawing a firm conclusion difficult. In addition, one patient imaged with HMDP in the study by Gallini et al was noted to have a Perugini grade 1 in the context of AL amyloid. ${ }^{7}$ Perhaps some degree of uptake of tracer uptake is to be expected in $\mathrm{AL}$ amyloid in light of the presence of microcalcifications at endomyocardial biopsy in this condition.

The absence of extra cardiac soft tissue uptake of PYP is an attractive property ${ }^{2}$ compared to DPD and HMDP. ${ }^{10,11}$ Retention of tracers in extra cardiac tissues may reduce cardiac retention even in the presence of significant cardiac ATTR amyloid. ${ }^{6}$ Although the use of SPECT may avoid false negatives due to lung uptake, SPECT will not resolve the relative paucity of cardiac tracer uptake due to its retention in visceral or skeletal muscle binding sites.

\section{WHOLE BODY OR CHEST PLANAR SCINTIGRAPHY OR SPECT/CT}

Gallini et al employed whole body planar scintigraphy rather than limiting their imaging to the chest. ${ }^{7}$ Our practice with ${ }^{99 \mathrm{~m}} \mathrm{Tc}$-PYP is to image only the chest with planar and SPECT/CT (Figure 1). Most ${ }^{99 \mathrm{~m}} \mathrm{Tc}-\mathrm{PYP}$ studies have used planar chest imaging and others have found anecdotally that whole body imaging for ${ }^{99 \mathrm{~m}} \mathrm{Tc}$ PYP does not provide additional data. ${ }^{2,12}$ In contrast, whole body planar scintigraphy is used in most studies with ${ }^{99 m}$ Tc-DPD perhaps as a result of extra cardiac soft tissue uptake with this tracer. ${ }^{13,14}$ Prior studies examining the utility of ${ }^{99 \mathrm{~m}} \mathrm{Tc}$-HMDP have employed whole body planar scintigraphy with or without SPECT imaging. ${ }^{7,15-17}$ SPECT can be useful to assess blood pool or rib uptake. CT may provide attenuation correction which is not possible with planar imaging alone. ${ }^{2}$ In cardiac centers, chest planar and cardiac SPECT/CT images would be a more familiar technique rather than whole body SPECT and a chest imaging approach for ${ }^{99 \mathrm{~m}} \mathrm{Tc}-$ PYP appears the most efficient.

\section{SEMI-QUANTITATIVE ANALYSIS}

Whether scintigraphy is limited to the chest or includes the whole body, visual grading by the Perugini method is possible. Similarly, whether imaging the whole body or the chest alone, it is also possible to perform semi-quantitative analysis. The utility of semiquantitative assessment using heart to body region ratios has been previously described. Galat et al from the Netherlands demonstrated that an early $(10 \mathrm{~min})$ heart to mediastinal ratio of $\geq 1.210$ for HMDP discriminated ATTR from AL cardiac amyloid with $100 \%$ sensitivity and specificity. ${ }^{15}$ These results were obtained from a cohort of 135 patients including 19 with AL amyloid and 74 with TTR amyloidosis. Gallini et al reported that a H/WB ratio of 3.28 had a $100 \%$ sensitivity and specificity for ATTR cardiac amyloid. ${ }^{7}$ Similarly, Perugini et al with ${ }^{99 \mathrm{~m}} \mathrm{Tc}-\mathrm{DPD}$ whole body imaging and Bokhari et al with ${ }^{99 \mathrm{~m}} \mathrm{Tc}$-PYP chest imaging have demonstrated excellent receiver operating characteristics for semi-quantitative ratios. ${ }^{4,18}$ In light of the excellent operating characteristic for $\mathrm{H} / \mathrm{CL}$ semi-quantitative ratios, whether whole body planar imaging with ${ }^{99 \mathrm{~m}} \mathrm{Tc}-\mathrm{PYP}$ to generate a H/WB semi-quantitative ratio (similar to that achieved by Gallini et al) might improve the diagnostic accuracy remains to be determined.

In addition to supporting the diagnosis of ATTR cardiac amyloid, semi-quantitative analysis also predicts prognosis. In contrast, the Perugini grading has failed to demonstrate prognostic significance. ${ }^{13}$ Currently the prognostic data supporting the utility of semi-quantitative analysis have been performed using PYP and DPD. ${ }^{12,14}$ In a multicenter US study which included 121 ATTR cardiac amyloid patients imaged with PYP-planar imaging, a H/CL ratio of $\geq 1.6$ was associated with decreased survival. Using a forward selection modeling technique, mortality at 5 years was calculated to be $67 \%$ vs $33 \%$ for those with or without an $\mathrm{H} / \mathrm{CL} \geq 1.6$ respectively. ${ }^{12}$ Although HMDP semi-quantitative analysis has not yet been shown to demonstrate prognostic significance, there are no theoretical reasons for it to be any less useful in this regard that PYP or DPD.

Since Perugini grades of 1-3 could represent AL amyloid, it is important to rule out plasma cell dyscrasias. The combination of (a) an absence of monoclonal protein and (b) detection of Perugini grade 2-3 had a reported $100 \%$ specificity for ATTR amyloid and was consistent across DPD, PYP, and HMDP imaging. ${ }^{5}$ The sensitivity of this algorithm varied and were 72, 70, and 57\% for PYP, DPD, and HMDP, respectively. The utility of a combined approach using 
an absence of monoclonal protein, early semi-quantitative ratio (H/CL for PYP), and Perugini grading is likely to be used in clinical practice.

\section{FUTURE DIRECTIONS}

Within nuclear cardiology, there has been significant interest in the use of PET tracers for imaging cardiac amyloid. ${ }^{2}$ Although PET will provide quantitative data as to tracer retention and possibly assessment of cardiac amyloid burden, the current novel PET tracers do not distinguish ATTR from AL amyloid. ${ }^{18} \mathrm{~F}$ sodium fluoride $(\mathrm{NaF})$ has demonstrated some utility in detecting ATTR but has not been compared with established ${ }^{99 \mathrm{~m}}$ Tc-labeled bone tracers. Imaging with ${ }^{99 \mathrm{~m}} \mathrm{Tc}$ scintigraphy promises to continue for the foreseeable future as the imaging test of choice to identify ATTR amyloid in suspected cases.

Nuclear imaging assessment of cardiac ATTR amyloid is not yet wrinkle-free. The work of Gallini et al provides further data on the potential for semiquantitative assessment to potentially improve the diagnostic accuracy of scintigraphy beyond that attained by visual grading alone. Previous collaborative multicentre work to write a diagnostic algorithm for ATTR amyloid had included visual grading and not semi-quantitative analysis. ${ }^{5}$ However, in recent ASNC and JNC publications, both visual grading and semi-quantitative analysis of planar scintigraphy images were encouraged and this reflects the additional diagnostic and prognostic data determined from semi-quantitative analysis. ${ }^{2,3}$ Semiquantitative analysis from planar scintigraphy will likely become more widely accepted as a part of the diagnostic algorithm and smooth out some of the remaining wrinkles in the accurate assessment of cardiac ATTR amyloid.

\section{Disclosure}

Dr. Small has nothing to disclose. Dr. Ruddy reports research grant support from GE Healthcare and Advanced Accelerator Applications.

\section{References}

1. Falk RH. Diagnosis and management of the cardiac amyloidoses. Circulation 2005;112(13):2047-60.

2. Singh V, Falk R, Di Carli MF, Kijewski M, Rapezzi C, Dorbala S. State-of-the-art radionuclide imaging in cardiac transthyretin amyloidosis. J Nucl Cardiol 2019;26(1):158-73.

3. Dorbala S, Bokhari S, Miller E, Bullock-Palmer R, Soman P, Thompson R. 99m Technetium-pyrophosphate imaging for transthyretin cardiac amyloidosis. ASNC Practice Points 2016.

4. Perugini E, Guidalotti PL, Salvi F, Cooke RM, Pettinato C, Riva $\mathrm{L}$, et al. Noninvasive etiologic diagnosis of cardiac amyloidosis using 99mTc-3,3-diphosphono-1,2-propanodicarboxylic acid scintigraphy. J Am Coll Cardiol 2005;46(6):1076-84.

5. Gillmore JD, Maurer MS, Falk RH, Merlini G, Damy T, Dispenzieri A, et al. Nonbiopsy diagnosis of cardiac transthyretin amyloidosis. Circulation 2016;133(24):2404-12.

6. Ross JC, Hutt DF, Burniston M, Page J, Steeden JA, Gillmore JD, et al. Quantitation of $(99 \mathrm{~m}) \mathrm{Tc}-\mathrm{DPD}$ uptake in patients with transthyretin-related cardiac amyloidosis. Amyloid 2018;25(3):203-10.

7. Gallini C, Tutino F, Martone R, Ciaccio A, Costanzo EN, Taborchi G, et al. Semi-quantitative indices of cardiac uptake in patients with suspected cardiac amyloidosis undergoing $99 \mathrm{mTc}-$ HMDP scintigraphy. J Nucl Cardiol 2019. https://doi.org/10.1007/ s12350-019-01643-w.

8. Rapezzi C, Gagliardi C, Milandri A. Analogies and disparities among scintigraphic bone tracers in the diagnosis of cardiac and non-cardiac ATTR amyloidosis. J Nucl Cardiol 2018. https://doi. org/10.1007/s12350-018-1235-6.

9. Stats MA, Stone JR. Varying levels of small microcalcifications and macrophages in ATTR and AL cardiac amyloidosis: implications for utilizing nuclear medicine studies to subtype amyloidosis. Cardiovasc Pathol 2016;25(5):413-7.

10. Hutt DF, Quigley AM, Page J, Hall ML, Burniston M, Gopaul D, et al. Utility and limitations of 3,3-diphosphono-1,2-propanodicarboxylic acid scintigraphy in systemic amyloidosis. Eur Heart $\mathbf{J}$ Cardiovasc Imaging 2014;15(11):1289-98.

11. Cappelli F, Gallini C, Costanzo EN, Tutino F, Ciaccio A, Vaggelli $\mathrm{L}$, et al. Lung uptake during $99 \mathrm{mTc}$-hydroxymethylene diphosphonate scintigraphy in patient with TTR cardiac amyloidosis: An underestimated phenomenon. Int J Cardiol 2018;254:346-50.

12. Castano A, Haq M, Narotsky DL, Goldsmith J, Weinberg RL, Morgenstern R, et al. Multicenter study of planar technetium 99m pyrophosphate cardiac imaging: predicting survival for patients with ATTR cardiac amyloidosis. JAMA Cardiol 2016;1(8):880-9.

13. Hutt DF, Fontana M, Burniston M, Quigley AM, Petrie A, Ross JC, et al. Prognostic utility of the Perugini grading of 99mTc-DPD scintigraphy in transthyretin (ATTR) amyloidosis and its relationship with skeletal muscle and soft tissue amyloid. Eur Heart J Cardiovasc Imaging 2017;18(12):1344-50.

14. Rapezzi C, Quarta CC, Guidalotti PL, Pettinato C, Fanti S, Leone $\mathrm{O}$, et al. Role of $(99 \mathrm{~m}) \mathrm{Tc}-\mathrm{DPD}$ scintigraphy in diagnosis and prognosis of hereditary transthyretin-related cardiac amyloidosis. JACC Cardiovasc Imaging 2011;4(6):659-70.

15. Galat A, Rosso J, Guellich A, Van Der Gucht A, Rappeneau S, Bodez D, et al. Usefulness of (99m)Tc-HMDP scintigraphy for the etiologic diagnosis and prognosis of cardiac amyloidosis. Amyloid 2015;22(4):210-20.

16. Cappelli F, Gallini C, Di MC, Costanzo EN, Vaggelli L, Tutino F, et al. Accuracy of $99 \mathrm{mTc}$-hydroxymethylene diphosphonate scintigraphy for diagnosis of transthyretin cardiac amyloidosis. J Nucl Cardiol 2019;26(2):497-504.

17. Glaudemans AW, van Rheenen RW, van den Berg MP, Noordzij $\mathrm{W}$, Koole M, Blokzijl $\mathrm{H}$, et al. Bone scintigraphy with (99m)technetium-hydroxymethylene diphosphonate allows early diagnosis of cardiac involvement in patients with transthyretinderived systemic amyloidosis. Amyloid 2014;21(1):35-44.

18. Bokhari S, Castano A, Pozniakoff T, Deslisle S, Latif F, Maurer MS. (99m)Tc-pyrophosphate scintigraphy for differentiating lightchain cardiac amyloidosis from the transthyretin-related familial and senile cardiac amyloidoses. Circ Cardiovasc Imaging 2013;6(2):195-201.

Publisher's Note Springer Nature remains neutral with regard to jurisdictional claims in published maps and institutional affiliations. 